\title{
Antimalarial Drug Resistance of Plasmodium falciparum in Africa: The Need for Novel Drug Treatments
}

\author{
Emmanuel Ebuka Elebesunu (iD 1, ${ }^{*}$, John Sunday Uhuo ${ }^{1}$ and Precious Ihechiluru Sylvanus ${ }^{1}$ \\ ${ }^{1}$ Department of Medical Laboratory Sciences, Enugu Campus, University of Nigeria, Enugu, Nigeria \\ "Corresponding author: Department of Medical Laboratory Sciences, Enugu Campus, University of Nigeria, Enugu, Nigeria. Tel: +234-8162815504, Email: \\ elebesunumichael@gmail.com
}

Received 2021 January 19; Accepted 2021 February 04.

Keywords: Antimalarial, Plasmodium falciparum, Drug Resistance, Africa, Treatment

\section{Dear editor,}

Malaria is a major public health threat and is one of the leading causes of death worldwide, with a death toll of over 400,000 annually (1). A great portion of these deaths comes from African countries, such as Burkina Faso, Niger, Mozambique, Tanzania, the Democratic Republic of Congo, and Nigeria, all accounting for about $51 \%$ of all malaria deaths worldwide (1). Over $90 \%$ of these malaria mortality cases is caused by Plasmodium falciparum, which is the most virulent form of the malaria parasite (1). In the past few decades, a major contributor to the increased severity and mortality rate of malaria is the nagging problem of drug resistance, mainly for P. falciparum, which has emerged against almost all used antimalarial drugs since the 1930 (2). This has greatly impeded global efforts to control malaria.

The major drugs initially used for the treatment of non-severe malaria were chloroquine (first-line treatment) and sulfadoxine-pyrimethamine (second-line treatment) (2). Chloroquine is considered as one of the most important drugs ever used in malaria treatment due to its costeffectiveness, ease of accessibility, low toxicity, as well as its high efficacy against malaria parasites. However, resistance to this drug emerged rapidly due to mutations that occurred in the $P$. falciparum chloroquine resistance transporter (Pfcrt) gene (2). This form of resistance was first recorded in Southeast Asia and soon spread globally to other continents, including Africa (2), and by 2010, most countries worldwide had stopped using chloroquine as a first-line antimalarial drug (2). The antifolate combination therapy using sulfadoxine and pyrimethamine was also widely used as an antimalarial drug due to its low cost and high efficacy. The drug consists of active agents that inhibit specific enzymes called dihydrofolate reductase
(DHFR) and dihydropteroate synthase (DHPS) (2), which are necessary for folate biosynthesis in the parasite; however, resistance has emerged due to multiple mutations in the genes that encode these enzymes (2). In Nigeria, this drug is still used as a prophylactic measure for the prevention of malaria in pregnant women, in line with the World Health Organization's recommendation of administering sulfadoxine-pyrimethamine (SP) as an intermittent preventive treatment (IPT) for expectant mothers in SubSaharan Africa (3). However, certain studies have shown an increasingly high level of resistance to the drug $(3,4)$, and the use of alternatives for malaria prevention in pregnancy are currently advised. Other antifolate combination therapies, like sulfalene-pyrimethamine and sulfamethoxazoletrimethoprim, have been used in P. falciparum malaria treatment, but resistance to these drugs has also emerged, just as with SP (5).

Some quinine derivatives, like amodiaquine, mefloquine, and halofantrine, have also been used in treating $P$. falciparum malaria, but they have either developed cross-resistance with chloroquine, which is also a quinine derivative, or they were found to have certain toxic effects on malaria-infected individuals (5). Resistance of $P$. falciparum to the quinine derivatives is due to mutations in the Pfmdr-1 gene; leading to resistance to the parasite (5). Atovaquone is another drug that has been used for the treatment of chloroquine-resistant falciparum malaria, but resistance to the drug quickly arose due to single point mutations in the cytochrome b gene of P.falciparum (5). Hence, it is currently used in combination with proguanil as an effective treatment for multidrug-resistant P. falciparum (5).

The WHO's recommendation of artemisinin-based combination therapies (ACTs) as choice drugs for treating uncomplicated malaria gave a ray of hope due to 
their potency against malaria parasites. These ACTs involve the combination of artemisinin derivatives (artemether, artesunate, and dihydroartemisinin) with other pharmacologically active compounds to increase efficacy and decrease the chances of drug resistance (6). The major ACTs currently recommended in most countries worldwide are artemether-lumefantrine (AL) and amodiaquine-artesunate (AQ-AS) (6). Despite their seemingly potent action, incidences of resistance to ACTs have been reported in certain regions of Southeast Asia6 and will most likely spread to other continents in a matter of years. A study conducted by Dama et al. in Mali showed the decreasing rates of $P$. falciparum susceptibility to artemether-lumefantrine treatment (7) and a more recent study by Uwimana et al. confirmed the emergence of mutant $P$. falciparum strains exhibiting artemisinin resistance in Rwanda (8). These studies proved that artemisininresistant strains have either spread from Southeast Asia to Africa or have emerged independently in Africa due to selective drug pressure. This emergence of drug resistance to ACTs is worrisome as it threatens global efforts to curb malaria and increases the possibility of $P$. falciparum malaria becoming untreatable.

In lieu of these, there is a great need for periodic testing of antimalarial susceptibility and also carrying out genetic surveillance of antimalarial resistance markers among $P$. falciparum strains in order to monitor and predict drug resistance patterns, with a view to effectively controlling the spread of antimalarial resistance. Numerous efforts have been made to develop a malaria vaccine, but no vaccine candidate so far has shown an appropriate level of efficacy in malaria prevention, mostly due to challenges associated with the parasite's complex life cycle and the antigenic variation at each stage of the life cycle (9). Hence, it is of utmost importance that novel drug targets are identified for the development of new drugs to effectively tackle the emerging problem of malaria multidrug resistance. Plasmodium proteases are a promising drug target due to their critical role in erythrocyte invasion and hemoglobin breakdown; thus, compounds, such as leupeptin, pepstatin, and fluoromethyl ketones that inhibit the cysteine, serine, and aspartic proteases are good antimalarial drug candidates as they have been effective in inhibiting the erythrocytic invasion and the growth and development of P. falciparum (10). Also, the inhibition of membrane transporters, like the plasmodial surface anion channel (PSAC) by anion transport blockers, such as phlorizin, dantrolene, furosemide, and niflumate have shown promising effects in inhibiting the nutrient transport of the intracellular parasite stage (10). Further research needs to be embarked on to establish the suitability of these compounds as modifiable and usable antimalarial agents in the nearest future.

\section{Footnotes}

Authors' Contribution: Emmanuel Ebuka Elebesunu developed and drafted the concept of this manuscript. Literature search and data collection were done by Emmanuel Ebuka Elebesunu and John Sunday Uhuo. Precious Ihechiluru Sylvanus carried out critical revision and language edits. All the authors have read and agreed with the final manuscript.

Conflict of Interests: The authors declare that they have no conflict of interests.

Funding/Support: The authors did not receive any funding for the research and preparation of this manuscript.

\section{References}

1. World Health Organization. World malaria report 2020. Geneva, Switzerland: World Health Organization; 2020.

2. Takala-Harrison S, Laufer MK. Antimalarial drug resistance in Africa: Key lessons for the future. Ann N Y Acad Sci. 2015;1342:627. doi: 10.1111/nyas.12766. [PubMed: 25891142]. [PubMed Central: PMC4527866].

3. Umar Y, Nasir I, Aliyu M, Dangana A, Vanstawa A. In-vitro antimalarial resistance pattern of Plasmodium falciparum infection among preg nant women in Northern Nigeria. Afro-Egyptian Journal of Infectious and Endemic Diseases. 2017;7(2):47-51. doi: 10.21608/aeji.2017.9170.

4. Agomo CO, Oyibo WA, Sutherland C, Hallet R, Oguike M. Assessment of markers of antimalarial drug resistance in Plasmodium falciparum isolates from pregnant women in Lagos, Nigeria. PLoS One. 2016;11(1). e0146908. doi: 10.1371/journal.pone.0146908. [PubMed: 26808627]. [PubMed Central: PMC4726598].

5. Bloland PB. Drug resistance in malaria. Geneva, Switzerland: World Health Organization; 2001.

6. Olasehinde GI, Diji-Geske RI, Fadina I, Arogundade D, Darby P, Adeleke A, et al. Epidemiology of Plasmodium falciparum infection and drug resistance markers in Ota Area, Southwestern Nigeria. Infect Drug Resist. 2019;12:1941-9. doi: 10.2147/IDR.S190386. [PubMed: 31308714] [PubMed Central: PMC6616117].

7. Dama S, Niangaly H, Ouattara A, Sagara I, Sissoko S, Traore OB, et al. Reduced ex vivo susceptibility of Plasmodium falciparum after oral artemether-lumefantrine treatment in Mali. Malar J. 2017;16(1):59. doi: 10.1186/s12936-017-1700-8. [PubMed: 28148267]. [PubMed Central: PMC5289056].

8. Uwimana A, Legrand E, Stokes BH, Ndikumana JM, Warsame M, Umulisa $\mathrm{N}$, et al. Emergence and clonal expansion of in vitro artemisinin-resistant Plasmodium falciparum kelch13 R561H mutant parasites in Rwanda. Nat Med.2020;26(10):1602-8. doi: 10.1038/s41591020-1005-2. [PubMed: 32747827]. [PubMed Central: PMC7541349].

9. Duffy PE, Patrick Gorres J. Malaria vaccines since 2000: Progress, priorities, products. NPJ Vaccines. 2020;5:48. doi: 10.1038/s41541-0200196-3. [PubMed: 32566259]. [PubMed Central: PMC7283239].

10. Belete TM. Recent progress in the development of new antimalarial drugs with novel targets. Drug Des Devel Ther. 2020;14:3875-89. doi: 10.2147/DDDT.S265602. [PubMed: 33061294]. [PubMed Central: PMC7519860]. 